\title{
GRB Prompt X-ray Emission
}

\author{
Takanori Sakamoto ${ }^{1,2,3}$ \\ ${ }^{1}$ Center for Research and Exploration in Space Science and Technology (CRESST), \\ NASA Goddard Space Flight Center, Greenbelt, MD 20771, U.S.A. \\ ${ }^{2}$ Joint Center for Astrophysics, University of Maryland, Baltimore County, \\ 1000 Hilltop Circle, Baltimore, MD 21250, U.S.A. \\ ${ }^{3}$ NASA Goddard Space Flight Center, Greenbelt, MD 20771, U.S.A. \\ email: Taka.Sakamoto@nasa.gov
}

\begin{abstract}
I present the observational properties of the prompt emission of supernova associated GRBs (SN-GRBs) focusing on temporal and spectral characteristics. I compare the properties of SN-GRBs with typical long GRBs to see whether there is a distinct difference or not. Furthermore, I present our attempt to search for hard X-ray emission prior to the discovery date from optically identified type Ibc supernovae using Swift BAT survey data.
\end{abstract}

Keywords. gamma rays: bursts

\section{Introduction}

After the first discovery of the supernova associated gamma-ray burst (SN-GRB) GRB 980425 detected by BATSE and BeppoSAX (Galama et al. 1998), the number of SN-GRBs has been increasing thanks to a rapid and accurate GRB position notice by HETE-2 (Ricker et al. 2003), INTEGRAL (Winkler et al. 2003), and Swift (Gehrels et al. 2004). On the other hand, we have started to see a large diversity in the characteristics of the prompt emission of SN-GRBs (e.g., Kaneko et al. 2007). I will review the current observational status of the prompt emission properties of spectroscopically identified SN-GRBs by comparing them to typical long GRBs.

\section{Sample of SN-GRBs}

Table 1 shows the list of the spectroscopically identified SN-GRB samples in this work. Three GRBs - 030329, 060218, and 100316D (bold fonts in the table) - have secure associations with supernovae since a spectral evolution from a non-thermal power-law to a supernova-like thermal feature is clearly seen in the optical spectroscopic observations (e.g., Stanek et al. 2003, Hjorth et al. 2003 for GRB 030329; e.g., Pian et al. 2006 for GRB 060218; e.g., Chornock et al. 2011 for GRB 100316D).

Table 1. Spectroscopically identified SN-GRB sample.

\begin{tabular}{|c|c|c|c|c|}
\hline GRB & SN & Mission & Redshift & SN-Type \\
\hline 980425 & $1998 \mathrm{bw}$ & BATSE/BSAX & 0.0085 & Ic \\
$\mathbf{0 3 0 3 2 9}$ & $2003 \mathrm{dh}$ & HETE-2 & 0.1685 & Ic \\
031203 & $2003 \mathrm{lw}$ & INTEGRAL & 0.1005 & Ibc \\
$\mathbf{0 6 0 2 1 8}$ & $2006 \mathrm{aj}$ & Swift & 0.0331 & Ic \\
091127 & $2009 \mathrm{nz}$ & Swift & 0.490 & Ic \\
$\mathbf{1 0 0 3 1 6 D}$ & $2010 \mathrm{bh}$ & Swift & 0.059 & Ic \\
$101219 \mathrm{~B}$ & $2010 \mathrm{ma}$ & Swift & 0.55 & Ic \\
\hline
\end{tabular}



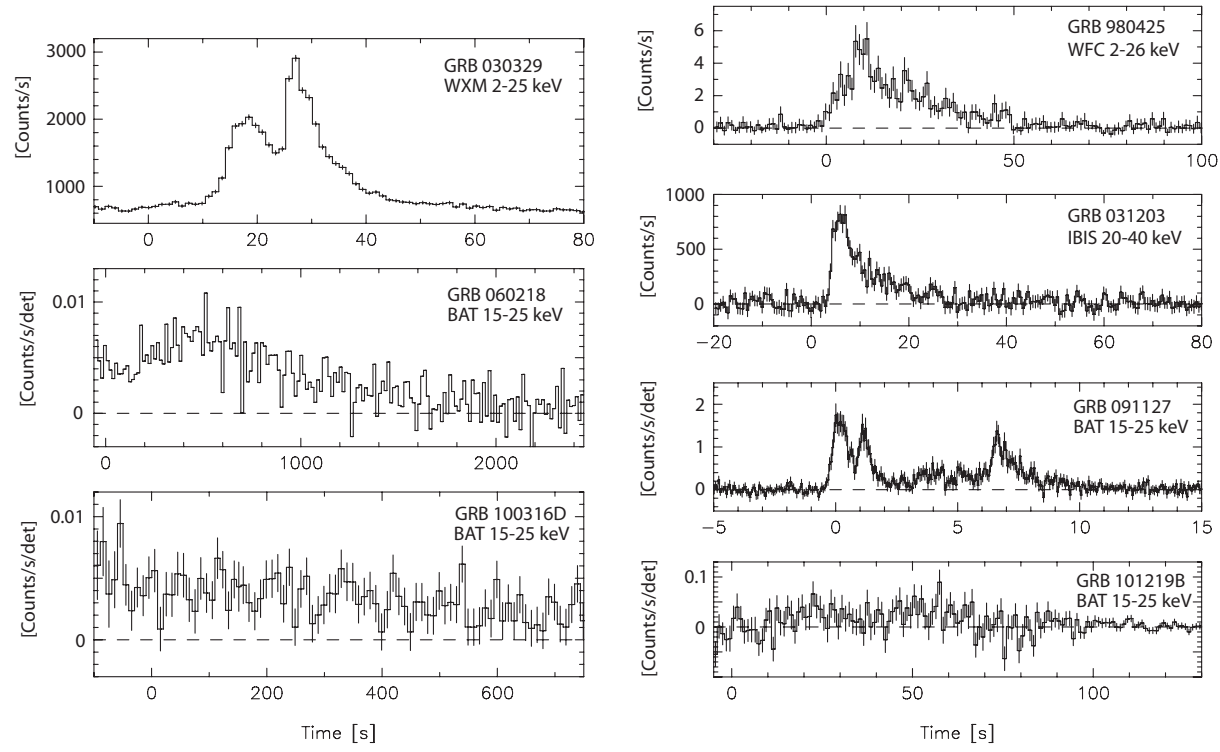

Figure 1. Prompt emission light curves of the SN-GRBs.

\section{Temporal properties}

\subsection{Light curves}

Figure 1 shows the prompt emission light curves of the SN-GRBs observed by various GRB instruments. As seen in the figure, there are large varieties in the light curves. For example, there are SN-GRBs which are composed of several bright overlapping pulses such as GRB 030329 and GRB 091127. On the other hand, GRB 060218, GRB 100316D and GRB 101219B show a very smooth and a long duration profile in their light curves.

\subsection{Durations}

The observed durations of SN-GRBs show diversity. While GRB 060218 and GRB 100316D show a duration of several thousands of seconds (Campana et al. 2006; Starling et al. 2011), SN-GRBs with a duration of a several tens to hundreds of seconds are also common. Although the durations of GRB 060218 and GRB 100316D are exceptionally long, the rest of the sample is well within the duration distribution of typical long GRBs (Figure 2).

\subsection{Lag-Luminosity relation}

Norris et al. (2000) found a correlation between the spectral lag and the peak luminosity (the so called lag-luminosity relation) of the prompt emission from the BATSE long GRBs. It is also known that short GRBs and several low luminous GRBs do not follow this relation (Norris \& Bonnell 2006). The lag-luminosity relation is further confirmed by the HETE-2 (Arimoto et al. 2010) and the Swift (Ukwatta et al. 2010) GRB samples. Figure 3 shows the BATSE, the HETE-2 and the Swift long GRBs and the SN-GRBs in a peak luminosity versus lag plane. The lag and the peak luminosity values of GRB 980425, GRB 031203 and GRB 060218 are extracted from Norris et al. (2000), Sazonov, Lutovinov \& Sunyaev (2004) and Liang et al. (2006), respectively. The values of the rest of the SN-GRBs are derived in this work. GRB 091127, GRB 030329 and possibly GRB 060218 are consistent with the lag-luminosity relations. According to Liang et al. (2006), the lag value of GRB 060218 has a large uncertainty since it requires an extrapolation 


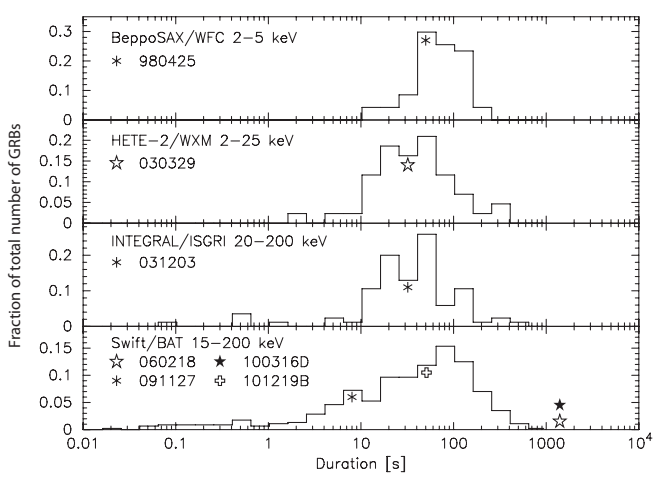

Figure 2. Observed duration distribution of BeppoSAX/WFC (2-5 $\mathrm{keV})$, HETE-2/WXM $(2-25 \mathrm{keV}), \quad I N-$ TEGRAL/ISGRI (20-200 keV) and Swift/BAT (15-200 keV) from top to bottom panel. The durations of the SN-GRBs are overlaid in the histograms.

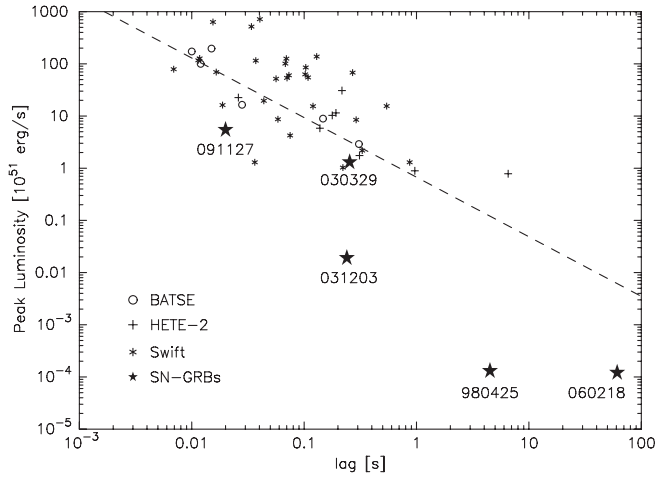

Figure 3. Lag-Luminosity relation of the BATSE (Norris et al. 2000), the HETE-2 (Arimoto et al. 2010) and the Swift (Ukwatta et al. 2010) long GRBs. The dotted line is the lag-luminosity relation, $L_{\text {iso }} \propto l a g^{-1.14}$, originally proposed by Norris et al. (2000). The SN-GRBs are shown as stars.

from the original lag measurement between the BAT (Barthelmy et al. 2005) and the XRT (Burrows et al. 2005) band to that of the BATSE standard band to be able to plot on the lag-luminosity plane. Based on their analysis, GRB 060218 is within the $2 \sigma$ confidence region of the relation. However, as noted by various previous works, GRB 980425 and GRB 031203 do not follow the relation. The relatively low peak luminosity for both GRB 980425 and GRB 031203 makes them outliers to this relationship. Note that since the low luminosity GRB 060218 seems consistent with the relation, not all low luminosity GRBs are outliers of the lag-luminosity relation.

\section{Spectral properties}

\subsection{Band function parameters}

The prompt emission spectra of SN-GRBs which have reported broad-band spectral parameters are well fitted with the Band function (Band et al. 1993). To compare the best fit parameters of the Band function between the SN-GRBs and typical long GRBs, the histograms of the low-energy photon index, $\alpha$, the high-energy photon index, $\beta$, and the spectral peak energy, $\mathrm{E}_{\text {peak }}$, of the long GRBs are shown in Figure $4 . \alpha$ and $\beta$ values

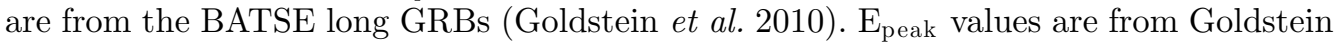
et al. (2010) for the BATSE, Sakamoto et al. (2005) and Pélangeon et al. (2008) for the HETE-2, and Sakamoto et al. (2011) for the Swift GRBs. The values of the best fit Band function parameters of the SN-GRBs are marked in the histograms. All the Band function parameters of the SN-GRBs are consistent with typical long GRBs.

\subsection{Additional blackbody component in X-ray spectrum}

The existence of an additional blackbody component in the prompt X-ray spectrum of GRB 060218 (Campana et al. 2006) and GRB 100316D (Starling et al. 2011) has been reported. The blackbody temperature is stable in the range of $0.1-0.2 \mathrm{keV}$ up to several hundreds of seconds after the trigger, and then, decreases to $<0.01 \mathrm{keV}$. I also found an additional blackbody component for GRB 101219B. The Swift XRT spectrum extracted from $152 \mathrm{~s}$ to $300 \mathrm{~s}$ after the trigger shows a significant improvement in the fit with a blackbody plus a power-law model $\left(\chi^{2} /\right.$ d.o.f. $\left.=85.8 / 100\right)$ over a power-law model 

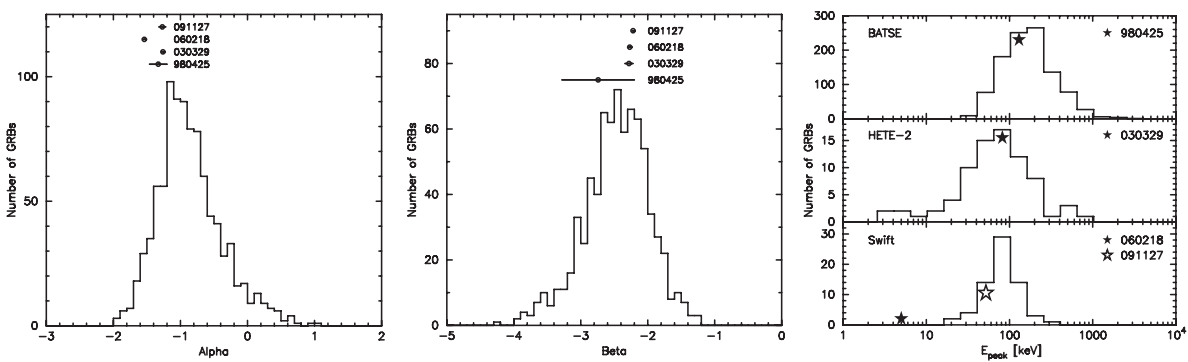

Figure 4. Distributions of the low-energy photon index $\alpha$ (left), the high-energy photon index $\beta$ (middle) and $\mathrm{E}_{\text {peak }}$ (right). The $\mathrm{E}_{\text {peak }}$ histograms of the BATSE, the HETE-2 and the Swift long GRBs are shown from top to bottom.

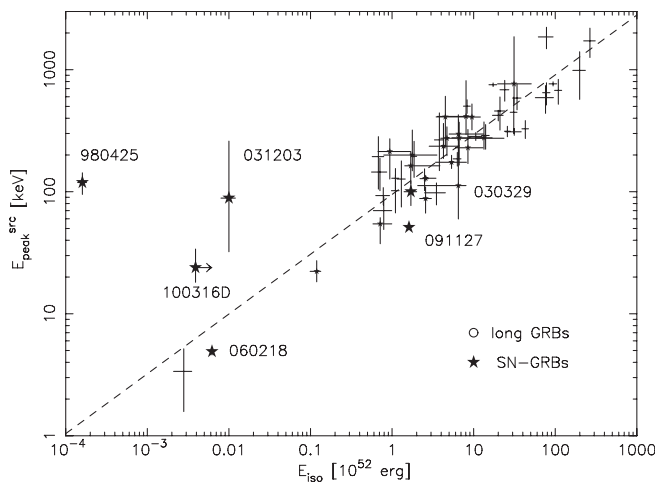

Figure 5. $\mathrm{E}_{\mathrm{peak}}-\mathrm{E}_{\mathrm{iso}}$ relation. The long GRB samples are from Amati et al. 2006 and Sakamoto et al. 2011.

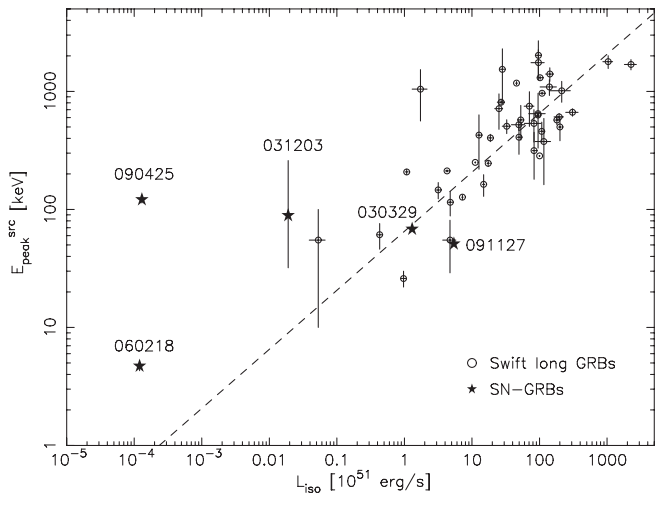

Figure 6. $\mathrm{E}_{\text {peak }}-\mathrm{L}_{\mathrm{iso}}$ relation. The long GRB samples are from Nava et al. 2012.

$\left(\chi^{2} /\right.$ d.o.f. $\left.=104.1 / 102\right)$. The F-test probability is $6.3 \times 10^{-5}$ between these two fits. The best fit blackbody temperature is $0.12 \pm 0.02 \mathrm{keV}$ which is similar to the case of GRB 060218 and GRB 100316D. Since most of the XRT spectra of long GRBs can well be fit by a simple power-law model, this additional blackbody component could be a unique spectral feature of SN-GRBs.

\section{3. $E_{\text {peak }}-E_{\text {iso }}$ and $E_{\text {peak }}-L_{\text {iso }}$ relations}

The empirical spectral relations between $\mathrm{E}_{\text {peak }}$ and the isotropically radiated $\gamma$-ray energy $\mathrm{E}_{\text {iso }}\left(\mathrm{E}_{\text {peak }}-\mathrm{E}_{\text {iso }}\right.$ relation; Amati et al. 2002) and between $\mathrm{E}_{\text {peak }}$ and the peak isotropic luminosity $\mathrm{L}_{\text {iso }}\left(\mathrm{E}_{\text {peak }}-\mathrm{L}_{\text {iso }}\right.$ relation; Yonetoku et al. 2004) are well discussed relationships of the prompt emission. As shown in Figure 5 and 6 , most of the long GRBs are consistent with the $\mathrm{E}_{\text {peak }}-\mathrm{E}_{\text {iso }}$ and the $\mathrm{E}_{\text {peak }}-\mathrm{L}_{\text {iso }}$ relations including the $\mathrm{SN}-\mathrm{GRBs}$ except GRB 980425 .

\section{Searching for hard X-ray emission in supernova}

We searched for hard X-ray emission using the Swift BAT survey data for 123 type Ibc and IIp supernovae $(<100 \mathrm{Mpc})$ discovered between 2005 and 2010. The BAT data starting from $\sim 28$ days prior to the discovery up to the discovery date are processed. No significant hard X-ray emission was found for ordinary type Ibc and IIp supernovae. Our result is consistent with a radio survey of ordinary type Ibc supernovae (Soderberg et al. 2006), and might indicate that a fundamentally different mechanism is required to produce a GRB from a supernova. 


\section{Summary}

- Burst duration, spectral lag and spectral parameters of SN-GRBs are consistent with typical long GRBs.

- In addition to the Band function spectrum, GRB 060218 and GRB 100316D (and possibly GRB 101219B) show $\mathrm{kT}=0.1-0.2 \mathrm{keV}$ blackbody component in the prompt emission spectrum.

- Most of the SN-GRBs follow the empirical relations between lag and luminosity, $\mathrm{E}_{\text {peak }}-\mathrm{E}_{\text {iso }}$ and $\mathrm{E}_{\text {peak }}-\mathrm{L}_{\text {iso }}$. However, GRB 980425 and possibly GRB 031203 are outliers of those relations.

- No hard X-ray emission is found from ordinary type Ibc and IIp supernovae.

\section{References}

Amati, L., et al. 2002, A\&A, 390, 81

Amati, L., et al. 2006, MNRAS, 372, 233

Arimoto, M., et al. 2010, PASJ, 62, 487

Band, D. L., et al. 1993, ApJ, 413, 281

Barthelmy, S. D., et al. 2005, Space Sci. Revs, 121, 143

Burrows, D. N., et al. 2005, Space Sci. Revs, 121, 165

Campana, S., et al. 2006, Nature, 442, 1008

Chornock, R., et al. 2011, ApJ submitted (astro-ph/arXiv:1004.2262)

Galama, T. J., et al. 1998, Nature, 434, 1104

Gehrels, N., et al. 2004, ApJ, 611, 1005

Goldstein, A., et al. 2010, http://gammaray.msfc.nasa.gov/ goldstein/

Hjorth, J., et al. 2003, Nature, 423, 847

Kaneko, Y., et al. 2007, ApJ, 654, 385

Liang, E.-W., et al. 2006, ApJ, 653, L81

Nava, L., et al. 2012, MNRAS, 421, 1256

Norris, J. P., et al. 2000, ApJ, 534, 248

Norris, J. P. \& Bonnell, J. T. 2006, ApJ, 643, 266

Pélangeon, A., et al. 2008, A\& $A, 491,157$

Pian, E., et al. 2006, Nature, 442, 1011

Ricker, G. R., et al. 2003, AIP-CP, 662, 3

Sakamoto, T. et al. 2005, ApJ, 629, 311

Sakamoto, T., et al. 2011, ApJS, 195, 1

Sazonov, S. Y., Lutovinov, A. A., \& Sunyaev, R. A. 2004, Nature, 430, 646

Soderberg, A. M., et al. 2006, ApJ, 638, 930

Starling, R. L. C., et al. 2011, MNRAS, 411, 2792

Stanek, K. Z., et al. 2003, ApJ, 591, L17

Ukwatta, T. N., et al. 2010, ApJ, 711, 1073

Winkler, C., et al. 2003, A\& A, 411, 1

Yonetoku, D., et al. 2004, ApJ, 609, 935

\section{Discussion}

Burrows: How does beaming affect your conclusions about the lack of hard X-ray emission from type Ibc SNe?

SAKАмото: We believe that beaming is playing a major role in generating a hard $\mathrm{X}$-ray prompt emission between SN-GRBs and ordinary type Ibc SNe. 
KULKARNI: As yourself noted the SN-GRB sample has strong selection biases. The events have to be at low redshift. This selection effect may strongly affect the detection of very soft (BB) components.

KULKARNI: It was my impression that the volumetric rate of SN-GRBs is an order of magnitude larger than those of cosmological GRBs. If so, the selection biases are even more severe. 